\title{
Malformación congénita de la vía aérea pulmonar: Reporte de un caso adulto
}

\author{
CARLOS ÁLVAREZ Z.*,**, CÉSAR CERDA C.****, \\ CARMEN CERDA A.*** y BELÉN SANHUEZA P.*****
}

\section{Congenital pulmonary airway malformation: An adult case report}

Congenital pulmonary airway malformation or CPAM (congenital cystic adenomatoid malformation) that involve the lung is an embryonic anomaly. CPAM is a rare condition in chilhood, and even more so in adulthood. In this article we report the case of a young adult man with CPAM and present a brief review about this condition. Case study: 23 years old man with several episodes of pneumonia and obstructive bronchial syndrome during his childhood. The patient complained of pleuritic chest pain, malaise and fever. On physical examination lung sound was diminished in the lower right zone of the chest, abundant coarse crackles were detected and there was dullness on percussion. Chest roentgenogram and computed tomography (CT) revealed a complex image of multiple cysts with air and fluid in the right lung. Because of the lack of response to medical treatment a right lower lobectomy was performed. Histopathology revealed a type 1 CPAM. After being discharged from the hospital the patient developed a broncho-pleural fistula, that was succesfully treated by a second surgical intervention. Three years after surgery the patient is free of symptoms. Discussion: CPAM is a rare congenital hamartomatous condition. This malformation in adults it is exceptional. Chest CT is the choice diagnostic technique. Considering the high neoplastic potential of this malformation, its treatment should be the surgical resection, even in asymptomatic patients.

Key words: Congenital pulmonary airway malformation, cystic adenomatoid malformation, Lung cyst, surgery.

\section{Resumen}

La malformación congénita de la vía aérea pulmonar (MCVAP), llamada también malformación congénita adenomatoidea quística que compromete al pulmón es una anomalía embrionaria. La MCVAP es de rara presentación en la infancia y es excepcional en los adultos. En este artículo comunicamos el caso de un hombre adulto con MCVAP y presentamos una breve revisión sobre esta condición. Caso clínico: Hombre de 23 años de edad con varios episodios de neumonía y de síndrome bronquial obstructivo en su niñez. El paciente se quejaba de dolor torácico pleurítico, malestar general y fiebre. En el examen físico se encontró disminución del murmullo pulmonar en la parte inferior del hemitórax derecho, abundantes crepitaciones y matidez. La radiografía y la tomografía computada de tórax (TC) revelaron múltiples imágenes hidro-aéreas complejas en el pulmón derecho. Debido a la falta de respuesta al tratamiento médico, se practicó una lobectomía inferior derecha. La histopatología demostró una MCVAP tipo 1. Después de ser dado de alta el paciente presentó una fistula broncopleural, la cual fue tratada exitosamente con una segunda intervención quirúrgica. Tres años después de la cirugía el paciente está asintomático. Discusión: La MCVAP es una malformación congénita hamartomatosa muy poco frecuente. En adultos es excepcional. La técnica diagnóstica de elección es la TC de tórax. Considerando el alto potencial neoplásico de esta malformación, su tratamiento debe ser la resección quirúrgica, incluso si el paciente está asintomático.

Palabras clave: Malformación congénita de la vía aérea pulmonar, malformación adenomataidea quística, quiste pulmonar, cirugía.

\footnotetext{
* Departamento de Cirugía, Facultad de Ciencias Médicas, Universidad de Santiago de Chile (USACH).

** Equipo de Cirugía de Tórax, Servicio de Cirugía, Complejo Asistencial Barros Luco (CABL).

*** Servicio de Anatomía Patológica, CABL.

**** Médico Becario de Cirugía, Universidad de Valparaíso.

***** Interno de Medicina, USACH.
} 


\section{Introducción}

La malformación congénita de la vía aérea pulmonar (MCVAP) es una anomalía poco frecuente del desarrollo embrionario, de causa desconocida y que suele presentarse como distrés respiratorio en neonatos. Se caracteriza por la presencia en el tejido pulmonar de múltiples masas poliquísticas ${ }^{1}$. El diagnóstico es raro en la infancia y excepcional en adultos, no habiendo reportes de estos últimos en Chile.

La MCVAP se encuentra dentro de las 4 principales lesiones congénitas quísticas del pulmón, junto con el secuestro pulmonar, el enfisema lobar congénito y los quistes pulmonares broncogénicos $^{2}$. En 1897, fue reconocida como una entidad distinta por Staerk ${ }^{3,4}$. En 1949, Chin y Tang, reportan el caso de un paciente con anasarca e introducen el término malformación adenomatoidea quística con el que se conoció durante muchos años la enfermedad ${ }^{5}$.

Reportamos el caso de un paciente de sexo masculino de 23 años con MCVAP del lóbulo pulmonar inferior derecho tratado por nosotros con éxito, y efectuamos una revisión actualizada del tema. Para la revisión bibliográfica se realizó una búsqueda a través del Catálogo Bello, LILACS, PubMed y SciELO sin restricción de tiempo o idioma.

\section{Caso clínico}

Paciente varón de 23 años de edad, con antecedentes de herniorrafia inguinal derecha a los 7 años. Refiere múltiples episodios de bronconeumonía y SBO durante la niñez. Consultó en la Unidad de Emergencia (UE) del Complejo Asistencial Barros Luco (CABL) el 31/10/2005 por dolor torácico derecho, compromiso del estado general y sensación febril no cuantificada.

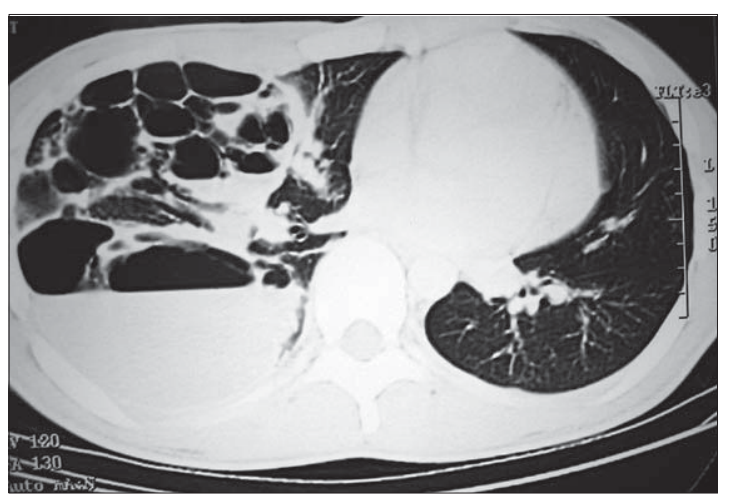

Figura 1. TC de tórax que muestra numerosos cavidades quísticas en el lóbulo inferior derecho, algunas más grandes con niveles hidroaéreos.
En el examen físico de ingreso el paciente estaba lúcido y orientado, con presión arterial y pulso normal, sin apremio respiratorio y con $38^{\circ} \mathrm{C}$ de temperatura axilar. Al examen físico segmentario destacaba en la auscultación de la base pulmonar derecha disminución del murmullo pulmonar, abundantes crepitaciones y matidez en la percusión. Se solicitó Rx de tórax y posteriormente TC de tórax, donde se observaron imágenes hidroaéreas complejas en hemitórax derecho compatibles con bronquiectasias, bulas y/o un quiste pulmonar infectado (Figura 1). Dentro de los exámenes de ingreso destacaba un recuento absoluto de leucocitos de $7,4 \times 10^{3} / \mu \mathrm{L}$, una proteína $\mathrm{C}$ reactiva de $324,4 \mathrm{mg} / \mathrm{L}$, gases en sangre arterial con una discreta alcalosis respiratoria $\left(\mathrm{pH}: 7,45 ; \mathrm{PO}_{2}: 86,2 \mathrm{mmHg}\right.$; saturación $\mathrm{O}_{2}: 97 \%$; $\mathrm{PCO}_{2}: 32,8 \mathrm{mmHg}$ : bicarbonato: 22, $0 \mathrm{mmol} / \mathrm{L}$; exceso de base: $-1,2 \mathrm{mmol} / \mathrm{L} ; \mathrm{CO}_{2}$ total: $23 \mathrm{mmol} / \mathrm{L}$ ). El paciente fue trasladado al Servicio de Medicina Interna donde se inició tratamiento antibiótico empírico con ceftriaxona más clindamicina y kinesioterapia respiratoria. Ante la falta de respuesta al tratamiento médico el caso fue presentado en reunión clínica de especialidad, en donde se decidió efectuar una lobectomía pulmonar inferior derecha de aseo. Fue operado el 21/11/2005, encontrándose múltiples quistes infectados y evidente pérdida de la arquitectura normal del parénquima pulmonar del lóbulo inferior derecho (LID), efectuándose la resección pulmonar planificada (Figura 2). En el postoperatorio evolucionó satisfactoriamente. Presentó mínimo enfisema subcutáneo, que en los días siguientes se reabsorbió rápidamente. Completó 7 días de tratamiento antibiótico. Estando en buenas condiciones generales y con control clínico y radiológico correcto, se dio de alta al sexto día de operado. Biopsia definitiva reveló: "MCVAP de LID tipo 1 de Stocker, con neumonías en distintas etapas de organización" (Figura 3). Tras 10 días del alta, paciente consultó nuevamente a la UE por percepción de enfisema subcutáneo. Se constató neumotórax derecho por lo que se re-hospitalizó. Se realizó TC de tórax que mostró alteraciones compatibles con colapso principalmente del lóbulo medio. Se efectuó entonces broncoscopia la que no logró resolver satisfactoriamente la atelectasia lobar. Fue trasladado al servicio de cirugía por sospecha de fístula broncopleural, realizándose nueva toracotomía, donde se encontró el lóbulo medio completamente colapsado y rotado sobre su eje y el lóbulo superior parcialmente expandido, se observó fístula bronco-pleural de pequeño bronquio en el lóbulo medio. Se realizó sutu- 
ra de fístula bronquial y se liberaron adherencias pleurales que mantenían al lóbulo medio en mala posición. En el postoperatorio evolucionó satisfactoriamente, sin filtración aérea y con total expansión pulmonar derecha. Se dio de alta al quinto día en perfectas condiciones. Posteriormente ha sido controlado en forma periódica, estando a más de 3 años de seguimiento, sin síntomas y con controles de imágenes que no evidencian enfermedad.

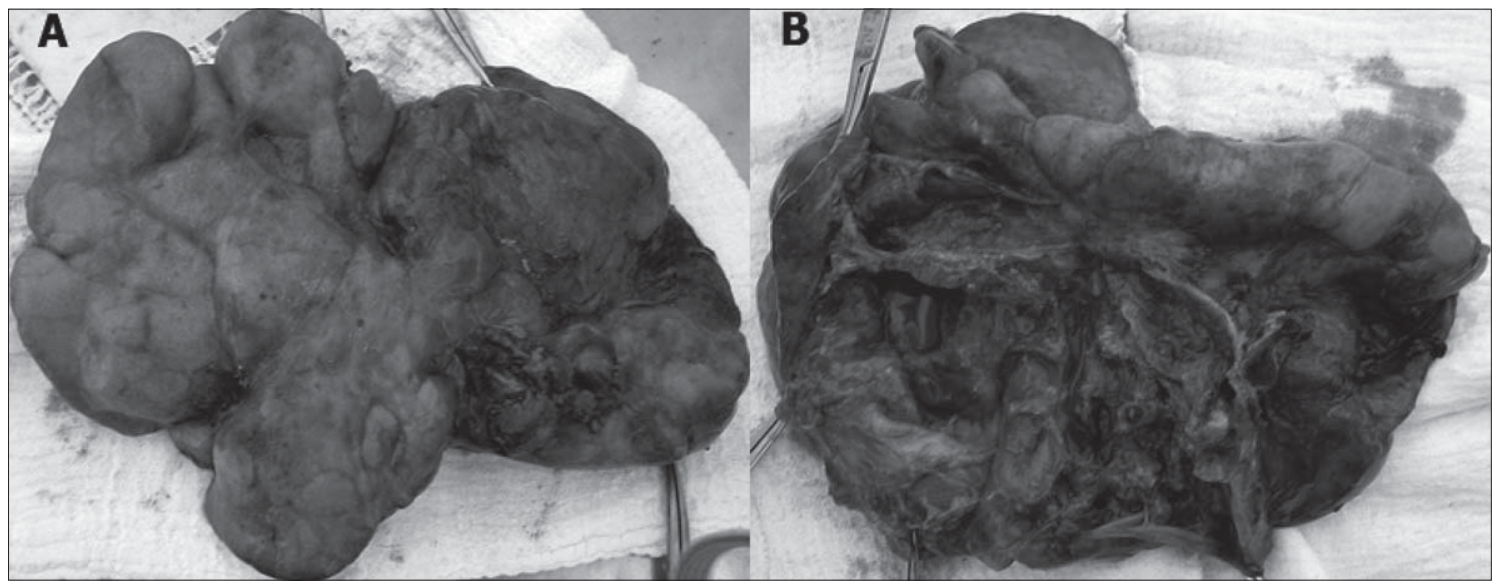

Figura 2. Pieza quirúrgica observándose (A) superficie pulmonar relativamente lisa y (B) parénquima pulmonar irregular con numerosas cavidades quísticas anfractuosas.
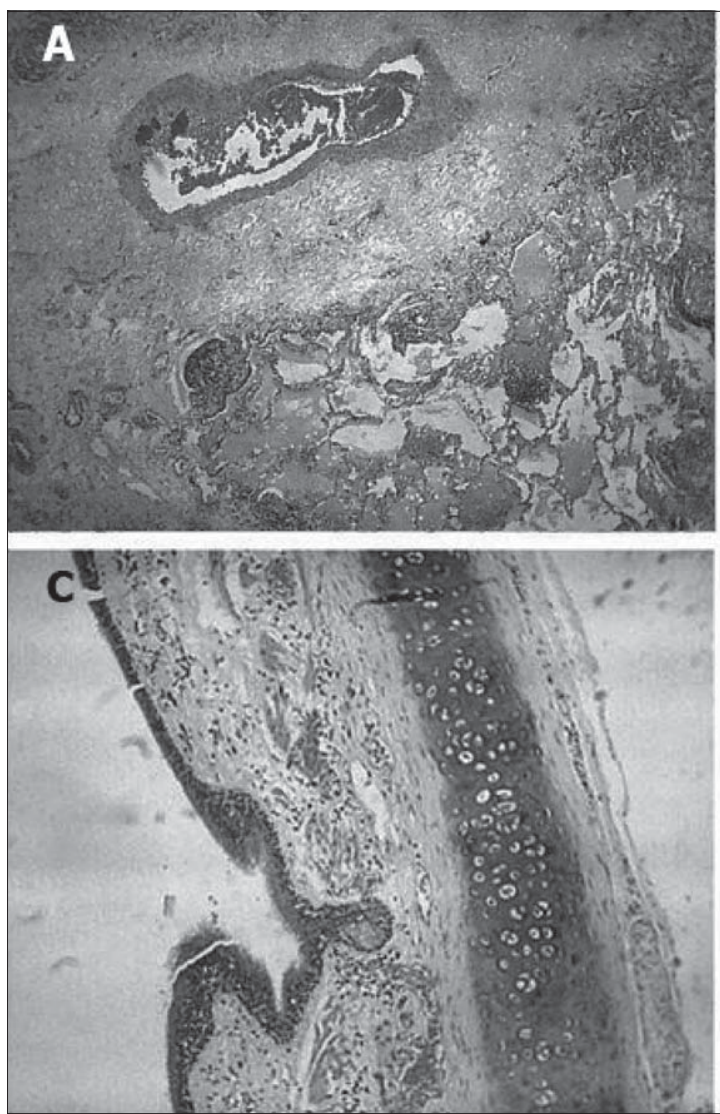
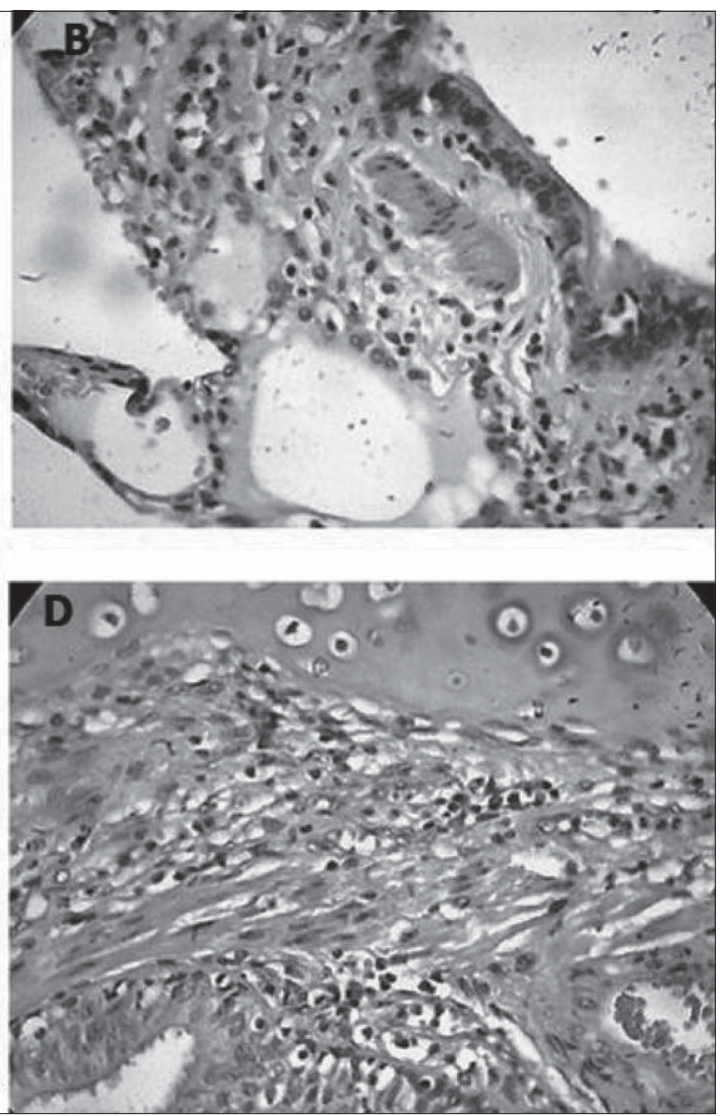

Figura 3. Corte histológico, hematoxilina-eosina 10x (A), panorámica de la lesión, con cavidades grandes, (B) 200x, la cavidad que aparece en el ángulo superior derecho, está revestida por epitelio cúbico pseudo-estratificado, ciliado. Un revestimiento similar, pero cilíndrico, aparece en el ángulo inferior izquierdo de la figura (D). Figuras (C) 100x y (D) 200x se observa cartílago en la pared de algunos quistes. En la figura (D) se aprecian fibras musculares lisas. Todo lo descrito es consistente con MCVAP tipo 1 de Stocker. 


\section{Discusión}

La MCVAP es una lesión pulmonar hamartomatosa, de carácter congénito y poco frecuente. Representa el $25 \%$ de todas las malformaciones congénitas pulmonares, se encuentra en 1 de cada 25.000 a 35.000 embarazos $^{6-8}$. La MCVAP probablemente resulte de la detención de la maduración bronquial y concomitante sobrecrecimiento de elementos mesenquimáticos, que van produciendo la apariencia adenomatoidea de la anomalía en los estadios precoces del desarrollo, esta área pseudotumoral con tejido pulmonar inmaduro habitualmente recibe circulación pulmonar. A menudo existen pequeñas comunicaciones bronquiales que llevan a la infección e inflamación de los quistes ${ }^{9-11}$.

La patogénesis de la MCVAP aún permanece desconocida. Mientras algunos investigadores han formulado la hipótesis de que resultaría de una falla en la interacción entre el endodermo y el mesodermo, otros sugieren un desbalance entre un incremento de la proliferación celular y un detrimento en la muerte celular programada (apoptosis). También se ha considerado que la MCVAP se produciría por una falla en el desarrollo vascular pulmonar, como se ha observado en otras malformaciones ${ }^{12-15}$.

En 1977 Stocker y cols, basados en la presentación clínica y las características patológicas relacionadas con la madurez del pulmón, desarrollaron una clasificación de la enferme$\mathrm{dad}^{16,17}$. Actualmente, este mismo autor la ha reformado y de tres tipos iniciales ha establecido cinco grupos (tipo 0 a 4) (Tabla 1). Nuestro caso corresponde al tipo 1, el más frecuente (> 65\%), presentando múltiples quistes pulmonares, el mayor de ellos de $8 \mathrm{~cm}^{17,18}$. La MCVAP generalmente afecta un lóbulo (tal como el caso que reportamos) y cuando es multilobar, sigue siendo unilateral. Todos los lóbulos se comprometen con igual frecuencia. Cuando las lesiones son bilobares o bilaterales, el pronóstico es malo debido a la hipoplasia del pulmón residual2, ${ }^{2,11}$.

El 83\% de los casos se pesquisa antes de los 6 meses de vida. La enfermedad se diagnostica con frecuencia en el período prenatal (18 a 24 semanas), gracias a la ecografía fetal ${ }^{19}$. Esto permite el tratamiento intrauterino o neonatal inmediato. La sintomatología más frecuente a esta edad es la dificultad respiratoria ${ }^{17,19}$. A veces está asociado a anasarca fetal, ascitis y polihidroamnios. No hay predilección por sexo o raza $^{20}$. La presentación en adultos es excepcional y habitualmente, al igual que en nuestro caso, existen antecedentes de infecciones respiratorias repetidas. No encontramos casos adultos publicados en la literatura nacional. Otras formas de inicio menos frecuentes reportadas en adultos son el neumotórax espontáneo, la bronquitis obstructiva y el dolor torácico. Excepcionalmente hay hemoptisis, un micetoma o un absceso pulmonar, ${ }^{4,17,19,21,22}$. Se han reportado casos de transformación maligna en carcinoma bronquioalveolar y rabdomiosarcoma embrionario pulmonar ${ }^{23-27}$.

Para la sospecha diagnóstica son fundamentales los exámenes de imágenes, aunque la forma adulta de la MCVAP muestra una amplia expresión radiológica, haciendo extremadamen-

Tabla 1. Clasificación anatomopatológica de Stocker de la malformación congénita de la vía aérea pulmonar ${ }^{31}$

\begin{tabular}{|c|c|c|c|c|c|}
\hline Hallazgo & Tipo 0 & Tipo 1 & Tipo 2 & Tipo 3 & Tipo 4 \\
\hline Frecuencia (\%) & $1-3$ & $>65$ & $10-15$ & 8 & $10-15$ \\
\hline $\begin{array}{l}\text { Tamaño máximo } \\
\text { del quiste }(\mathrm{cm})\end{array}$ & 0,5 & 10 & 2,5 & 1,5 & 7 \\
\hline Epitelio & $\begin{array}{c}\text { Ciliado, } \\
\text { pseudoestratificado, } \\
\text { columnar alto, con } \\
\text { células globosas }\end{array}$ & $\begin{array}{c}\text { Ciliado, } \\
\text { pseudoestratificado, } \\
\text { columnar alto }\end{array}$ & $\begin{array}{l}\text { Ciliado, } \\
\text { cuboidal } \\
\text { o columnar }\end{array}$ & $\begin{array}{l}\text { Ciliado, } \\
\text { cuboidal }\end{array}$ & $\begin{array}{l}\text { Lineal, alveolar } \\
\text { aplanado }\end{array}$ \\
\hline $\begin{array}{l}\text { Espesor del } \\
\text { músculo liso en } \\
\text { los quistes }(\mu \mathrm{m})\end{array}$ & $100-500$ & $100-300$ & $50-100$ & $0-50$ & $25-100$ \\
\hline Células mucosas & $100 \%$ de los casos & $33 \%$ de los casos & Ausente & Ausente & Ausente \\
\hline Cartílago & $100 \%$ de los casos & $5-10 \%$ de los casos & Ausente & Ausente & Raro \\
\hline Músculo estriado & Ausente & Ausente & $5 \%$ de los casos & Ausente & Ausente \\
\hline
\end{tabular}


te difícil el diagnóstico preoperatorio. En la Rx de tórax se ven alteraciones que varían según la forma de presentación, pudiendo observarse imágenes de neumonía, derrame pleural, neumotórax o lesiones quísticas, que plantean el diagnóstico diferencial con el quiste broncogénico, el secuestro pulmonar, los neumatoceles, las bronquiectasias, y aún las hernias diafragmáticas, en las que las asas enrolladas en el tórax pueden simular estructuras quísticas ${ }^{17,21}$. En la TC de tórax se observa neumonía asociada a una masa quística compleja. En el adulto puede simular una neoplasia. Los quistes pueden ser único o múltiples, conteniendo aire, líquido o ambos. Estas masas quísticas se suelen reforzar heterogéneamente con el contraste $6,17,19$. En nuestro caso se observaron imágenes hidroaéreas complejas en hemitórax derecho sugerentes de bronquiectasias, bulas $\mathrm{y} / \mathrm{o}$ un quiste pulmonar infectado. La TC permite valorar adecuadamente la extensión de la lesión y las condiciones del pulmón contralateral, lo que facilita una mejor aproximación operatoria. El tratamiento es la resección de las lesiones, aún para los pacientes asintomáticos, con el fin de prevenir la infección recurrente y por su potencial transformación neoplásica, incluso en la primera infancia ${ }^{24}$. La cirugía de elección es la lobectomía, aunque puede ser necesario realizar una resección más extensa cuando la enfermedad afecta más de un lóbulo. Sin embargo, ante la disyuntiva de una neumonectomía la resección segmentaria reglada o atípica puede ser una alternativa en pacientes bien seleccionados ${ }^{28,29}$. El abordaje inicial puede ser por videotoracoscopia, no obstante, se requiere de gran experiencia para el uso de esta técnica, con la cual es factible obtener la exéresis completa evitando los inconvenientes de la toracotomía. Sin embargo, es posible que las adherencias pleurales o la fibrosis hiliar impidan el procedimiento por esta aproximación ${ }^{30}$. La presencia de tejido residual tras la intervención puede favorecer la presencia de infecciones repetidas y la transformación maligna; por ello, es importante el seguimiento clínico y con imágenes de estos enfermos ${ }^{17,19}$.

\section{Conclusión}

La MCVAP es una rara malformación congénita que se puede diagnosticar en adultos. A pesar de ello debiera ser considerada en los enfermos con infecciones pulmonares recurrentes, neumotórax, o cuando se presentan infiltrados pulmonares que no se resuelvan sin otra causa aparente. Frente a estos hallazgos la TC de tórax es el examen de elección para una correcta evaluación. El tratamiento es la resección pulmonar incluso en asintomáticos debido a su demostrada capacidad de malignizarse.

\section{Bibliografía}

1.- MILLER R K, SIEBER W K, YUNIS E J. Congenital adenomatoid malformation of the lung. A report of 17 cases and review of the literature. Pathol Annu 1980; 15 (Pt 1): 387-402.

2.- TAKEDA S, MIYOSHI S, INOUE M, OMORI K, OKUMURA M, YOON H E, et al. Clinical spectrum of congenital cystic disease of the lung in children. Eur J Cardiothorac Surg 1999; 15: 11-7.

3.- KWITTKEN J, REINER L. Congenital cystic adenomatoid malformation of the lung. Pediatrics 1962; 30: 759-68.

4.- $\quad$ PLIT M L, BLOTT J A, LAKIS N, MURRAY J, PLIT M. Clinical, radiographic and lung function features of diffuse congenital cystic adenomatoid malformation of the lung in an adult. Eur Respir J 1997; 10: 16802.

5.- CH'N K Y, TANG M Y. Congenital adenomatoid malformation of one lobe of a lung with general anasarca. Arch Pathol 1949; 48: 221-9.

6.- ZYLAK C J, EYLER W R, SPIZARNY D L, STONE C H. Developmental lung anomalies in the adult: radiologic-pathologic correlation. Radiographics 2002; 22 Spec No: S25-43.

7.- SÁNCHEZ ABUÍN A, SOMOZA I, LIRAS J, MÉNDEZ $\mathrm{R}$, TELLADO M, RÍOS J, et al. Congenital cystic adenomatoid malformation associated with pulmonary sequestration. Cir Pediatr 2005; 18: 39-41.

8.- LABERGE J M, FLAGEOLE H, PUGASH D, KHALIFE S, BLAIR G, FILIATRAULT D, et al. Outcome of the prenatally diagnosed congenital cystic adenomatoid lung malformation: a Canadian experience. Fetal Diagn Ther 2001; 16: 178-86.

9.- BOGERS A J, HAZEBROEK F W, MOLENAAR J, BOS E. Surgical treatment of congenital bronchopulmonary disease in children. Eur J Cardiothorac Surg 1993; 7: 117-20.

10.- SCHNEIDER J R, ST CYR J A, THOMPSON T R, JOHNSON D E, BURKE B A, FOKER J E. The changing spectrum of cystic pulmonary lesions requiring surgical resection in infants. J Thorac Cardiovasc Surg 1985; 89: 332-9.

11.- BAILEY P V, TRACY T J R, CONNORS R H, DEMELLO D, LEWIS J E, WEBER T R. Congenital bronchopulmonary malformations. Diagnostic and therapeutic considerations. J Thorac Cardiovasc Surg 1990; 99: 597-602.

12.- CARDOSO W V, ITOH A, NOGAWA H, MASON I, BRODY J S. FGF-1 and FGF-7 induce distinct patterns 
of growth and differentiation in embryonic lung epithelium. Dev Dyn 1997; 208: 398-405.

13.- CASS D L, QUINN T M, YANG E Y, LIECHTY K W, CROMBLEHOLME T M, FLAKE A W, et al. Increased cell proliferation and decreased apoptosis characterize congenital cystic adenomatoid malformation of the lung. J Pediatr Surg 1998; 33: 1043-6; discussion 1047.

14.- CLOUTIER M M, SCHAEFFER D A, HIGHT D. Congenital cystic adenomatoid malformation. Chest 1993; 103: 761-4.

15.- MAS A, MIRAPEIX R M, DOMINGO C, SAÑUDO J R, TORREMORELL M D, MARÍN A. Pulmonary hypoplasia presented in adulthood as a chronic respiratory failure: report of two cases. Embryology, clinical symptoms and diagnostic procedures. Respiration 1997; 64: 240-3.

16.- STOCKER J T, MADEWELL J E, DRAKE R M. Congenital cystic adenomatoid malformation of the lung. Classification and morphologic spectrum. Hum Pathol 1977; 8: 155-71.

17.- RUPÉREZ PEÑA S, RUIZ DEL PRADO M Y, AROSTEGI KAREAGA N, GARCÍA DE ANDOIN BARANDIARÁN N, ITURRIOZ MATA A, ALONSO

T L. Cystic adenomatoid malformation: late diagnosis of a congenital anomaly. An Pediatr (Barc) 2003; 59: 396-9.

18.- PHAM T T, BENIRSCHKE K, MASLIAH E, STOCKER J T, YI E S. Congenital pulmonary airway malformation (congenital cystic adenomatoid malformation) with multiple extrapulmonary anomalies: autopsy report of a fetus at 19 weeks of gestation. Pediatr Dev Pathol 2004; 7: 661-6.

19.- LUJAN M, BOSQUE M, MIRAPIX R M, MARCO M T, ASCENSIO O, DOMINGO C. Late-onset congenital cystic adenomatoid malformation of the lung. Embriology, clinical sinptomatology, diagnostic procedure, therapeutic approach and clinical follow-up. Respiration 2002; 69: 148-54.

20.- DI GIORGIO A, AL MANSOUR M, CARDINI C L, FONSI G B, CARMENINI G, GUELI N. Congenital cystic adenomatoid malformation of the lung presenting as pyopneumothorax in an eighteen-year-old woman. J Thorac Cardiovasc Surg 2001; 122: 1034-6.

21.- HEIJ H A, EKKELKAMP S, VOS A. Diagnosis of congenital cystic adenomatoid malformation of the lung in newborn infants and children. Thorax 1990; 45: $122-5$.

22.- CAY A, SARIHAN H. Congenital malformation of the lung. J Cardiovasc Surg (Torino) 2000; 41: 50710

23.- DE PERROT M, PACHE J C, SPILIOPOULOS A. Carcinoma arising in congenital lung cysts. Thorac Cardiovasc Surg 2001; 49: 184-5.

24.- GRANATA C, GAMBINI C, BALDUCCI T, TOMA P, MICHELAZZI A, CONTE M, et al. Bronchioloalveolar carcinoma arising in congenital cystic adenomatoid malformation in a child: a case report and review on malignancies originating in congenital cystic adenomatoid malformation. Pediatr Pulmonol 1998; 25: 626.

25.- WEST D, NICHOLSON A G, COLQUHOUN I, POLLOCK J. Bronchioloalveolar carcinoma in congenital cystic adenomatoid malformation of lung. Ann Thorac Surg 2007; 83: 687-9.

26.- UEDA K, GRUPPO R, UNGER F, MARTIN L, BOVE $\mathrm{K}$. Rhabdomyosarcoma of lung arising in congenital cystic adenomatoid malformation. Cancer 1977; 40: 383-8.

27.- DOLADZAS T, ARVELAKIS A, KARAVOKYROS I G, GOUGOUdI E, PIKOULIS E, PATSOURIS E, et al. Primary rhabdomyosarcoma of the lung arising over cystic pulmonary adenomatoid malformation. Pediatr Hematol Oncol 2005; 22: 525-9.

28.- KIM H K, CHOI Y S, KIM K, SHIM Y M, KU G W, AHN K M, et al. Treatment of congenital cystic adenomatoid malformation: should lobectomy always be performed? Ann Thorac Surg 2008; 86: 249-53.

29.- FIBLA ALFARA J J, MOLINS LÓPEZ-RODÓ L, MIER ODRIOZOLA J M. Malformación adenomatoidea quística pulmonar y de afectación bilateral en el adulto. Arch Bronconeumol 2008; 44: 226.

30.- CONGREGADO M, LOSCERTALES J, GIRÓNARJONA J C, JIMÉNEZ-MERCHÁN R, ARROYOTRISTÁN A, GONZÁLEZ CÁMPORA R. Tres casos de malformación adenomatoidea quística en el adulto tratados por cirugía videotoracoscópica. Arch Bronconeumol 2004; 40: 236-9.

31.- DAIL D H, HAMMER S P. Pulmonary Pathology. $3^{\mathrm{a}}$ Edición Springer Verlag. New York 2008; pág 157.

\footnotetext{
Correspondencia a:

Dr. Carlos Álvarez Zepeda

Salmos Sur 107, Las Palmas de Maipú.

Maipú, Santiago, Chile

Fono/fax: 551-4320

E-mail: doctorcmaz@gmail.com
} 